\title{
Tensile behaviour of natural fibres. Effect of loading rate, temperature and humidity on the "accommodation" phenomena
}

\author{
V. Placet $^{1, \mathrm{a}}$ \\ ${ }^{1}$ Department of Applied Mechanics - FEMTO-ST Institute - UMR CNRS 6174 - University of \\ Franche-Comté - 25000 Besançon, France
}

\begin{abstract}
The use of natural fibres in high performance composite requires an accurate understanding of the mechanical behaviour of the fibres themselves. As for all biobased materials, the mechanical properties of natural fibres depend generally on the testing rate and on the environmental conditions. In addition, natural fibres as hemp for example exhibit a particular mechanism of stiffness increase and accommodation phenomena under cyclic loading. Loading rate, temperature and humidity effects on the viscoelastic properties of hemp fibres were investigated in this work. The collected results clearly emphasis the involvement of time-dependant and mechano-sorptive mechanisms.
\end{abstract}

\section{Introduction}

Natural fibres are considered to be a potential alternative to glass fibres in composites applications. To practical purposes, environmental awareness and an increasing concern with energy crisis have stimulated many industrial fields to look for sustainable materials replacing conventional synthetic materials. Natural fibres such as hemp, flax and sisal have been identified as attractive candidates. They are cheap, abundant and renewable, and have good specific properties. The use of natural fibres in high performance composite requires an accurate understanding of the mechanical behaviour of the fibres themselves. Only a limited number of studies have been conducted on the tensile behaviour of natural fibres. The review of literature shows a large discrepancy in reported tensile strength and Young's modulus of natural fibres. As for all biobased materials, the mechanical properties of natural fibres depend generally on the temperature [1,2], the humidity [3], the testing duration and rate [1]. The strength usually increases with moisture content and decreases with temperature, whereas the Young's modulus decreases as water is absorbed. In addition, the determination of the tensile properties of natural fibres involves some additional problems due to the variations in the fibre diameter, the influence of the clamping length, the measurement of the elongation, the clamping effects..., and also depends on the equipment used for tensile testing, the methodology used to measure the fibre's cross-sectional area and the methodology used to compute Young's modulus. So, the investigation of the tensile behaviour of natural fibres is not so trivial.

\footnotetext{
a e-mail : vincent.placet@univ-fomte.fr
} 
A really particular behaviour has been also underlined by quite a few authors [4-7]: under cyclic loading conditions, the longitudinal tensile Young's modulus of natural fibres increases according to the number of cycles. Contrary to all expectations, periodic stress did not lead to fatigue of the material but, quite to the contrary, to an increase in the fibre's rigidity. Baley [4] noticed an increase of $60-80 \%$ on flax fibres. It is also clear that the rigidity of the fibre increases until a constant value is reached, after a certain number of cycles, suggesting the presence of an "accommodation" phenomenon [7]. The explanation for this complex and unforeseen mechanical behaviour certainly lies in the macromolecular organisation of the cell wall. We recall that the mechanical strength of natural fibres is mostly provided by the $S_{2}$ layer of the cell wall in the longitudinal direction, in particular by the crystalline cellulose microfibrils which are spirally wound in a matrix of amorphous hemicellulose and lignin. These microfibrils are tilted by an angle of the order of $10^{\circ}$ with respect to the axis of the fibre. The increase in rigidity of the fibre resulting from cyclic stresses could thus be attributed to several mechanisms. At the level of the microfibrils, the longitudinal tension in the fibres could involve not only tensional stresses, but also torsion effects. This torsion stress, which initially impedes the tension, could subside or relax as a result of repeated stress. It is also probable that rearrangements and re-orientations of the cellulose microfibrils [4] and/or changes in the cristallinity fraction occur in the fibres. The hypothesis of collapse of the weak primary cell walls, and delamination between cells [5] can also be envisaged. Only macromolecular investigations could allow the above hypothesis to be verified.

In this work, we propose to investigate the effect of the temperature, the loading rate, and the humidity on the stiffering phenomenon under cyclic loading of hemp fibres (Cannabis sativa L.).

\section{Material and method}

\subsection{Natural fibres}

The hemp fibres (Cannabis sativa L.) tested were procured from the LCDA Company in France. They were delivered in a jumbled state. Elementary hemp fibres were isolated by hand from the initial bundles. According to the ASTM standard D3379 and also to simplify handling of the fibres, thin paper with glue was used as mounting tabs. Fibres are positioned on a self-adhesive paper frame with a window. A drop of glue is added to each extremity and the paper frame then clamped onto the testing machine. Before the beginning of each test, the paper frame is cut.

\subsection{Experimental set up}

The characterization of the mechanical behaviour of unitarian fibres (tubular elements of some tens microns in diameter and a few millimetres in length) under thermal andlor hydric control is not straightforward. The literature underlines these difficulties [8]. By this way, for this work, a specific experimental set up was developed. A Dynamic Mechanical Analyser (Bose Electroforce 3230) was implemented with a thermal chamber and a humidity generator to achieve the control of the environment around the samples. This apparatus allows quasi-static investigations as harmonic tests to be performed. This instrument employs a moving magnet linear motor to apply the solicitations to the sample. The applied force is measured with a load sensor of $2 \mathrm{~N}$ with a resolution of about $1 \mathrm{mN}$, and the displacement is measured using a LVDT with a resolution of $1 \mu \mathrm{m}$. The Relative Humidity Generator (HumiSys HF from Instruquest Inc.) is designed to inject humidity inside a sample chamber using a heated transfer line with a maximum flow rate of $5 \mathrm{~L} / \mathrm{min}$. The range of humidity is $2 \%-97 \%$ with a maximum temperature of saturator of $75^{\circ} \mathrm{C}$. This apparatus is versatile and allows an automatic Relative Humidity (RH) control in the sample chamber using a temperature and a humidity sensors placed inside the chamber, a few centimetres from the sample.

Usually, the Dynamical Mechanical testing provides a method for determining elastic and loss moduli as a function of temperature, frequency or time or both. DMA is commonly used to localise 
the transition regions, using rapid moduli change in plots of modulus versus temperature. This method can also be used to evaluate the degradation of the material properties according to time or environment. This technique has been already used successfully to study the hygrothermal degradation of wood [9-10]. It consists in measuring the evolution of the viscoelastic properties according to time at constant temperature and humidity and to relate the evolution of the viscoelastic properties to the mechanical degradation or rearrangements of material during tests.

Fibres were submitted to cyclic loading at around 10 to $15 \%$ of the stress at rupture (which involves an amplitude $\mathrm{Pk}-\mathrm{Pk}$ of loading around $50 \mathrm{mN}$ and a displacement around $15 \mu \mathrm{m}$ ). The clamping length is about $10 \mathrm{~mm}$.

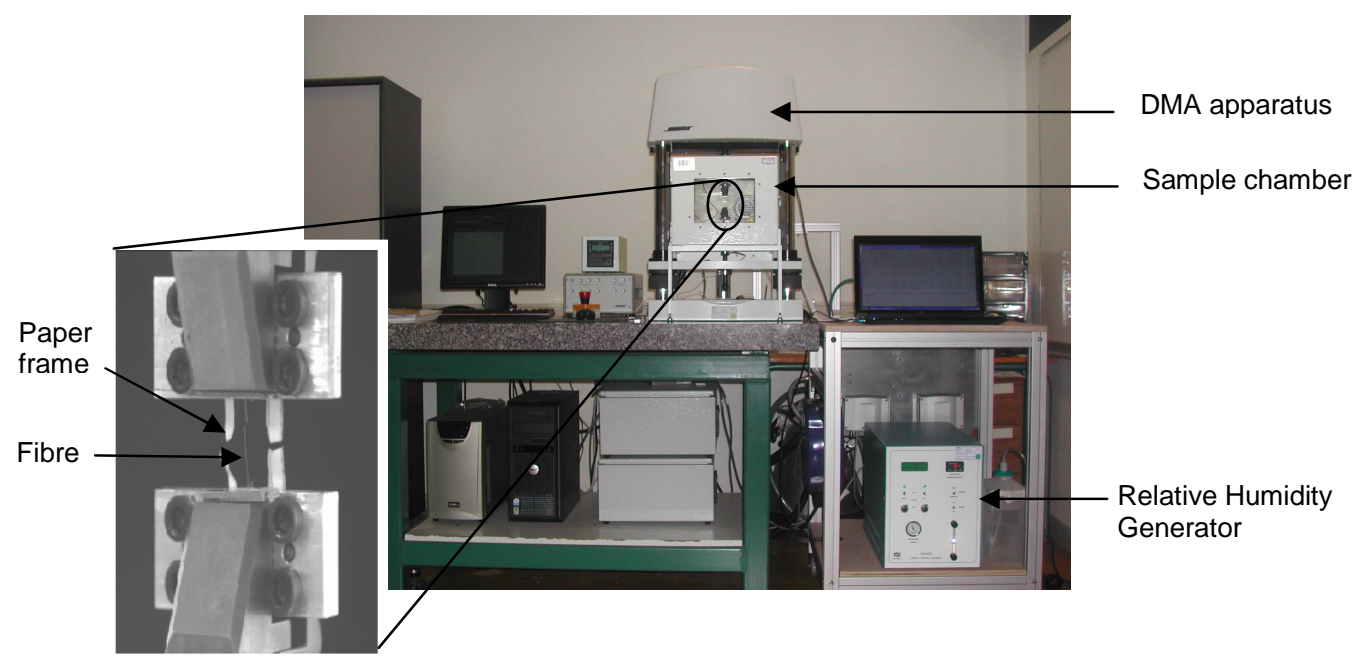

Natural fibre in clamps

Fig. 1. Experimental set-up

Three types of tests were performed. For each test, the viscoelastic properties of the fibre are measured according to time with regular interval. Three samples were analysed in order to check the repeatability of the measurements.

During the first series of tests, the fibres were tested at different plateau temperatures $\left(25^{\circ} \mathrm{C}, 100^{\circ} \mathrm{C}\right.$, $150^{\circ} \mathrm{C}, 200^{\circ} \mathrm{C}$ and $220^{\circ} \mathrm{C}$ ), at a frequency of $1 \mathrm{~Hz}$, for a maximum duration of 16 hours. The second series of tests were performed at room temperature at different frequencies $(0.01$ to $1 \mathrm{~Hz})$ and the last ones at room temperature with humidity control (from 5\% to 95\%).

\section{Results and discussion}

To avoid any mistake in the interpretation of the results (due to the method of determination of the tensile properties and due to the natural discrepancies of biological materials), data are presented using the normalized value of the viscoelastic properties.

Contrary to all expectations, periodic stress did not lead to a decrease in its mechanical properties but, quite to the contrary, to an increase in the fibre's rigidity. In Figure 2, it can be clearly seen that the rigidity of the fibre increases until, after 60000 cycles, it is 1.6 times greater than its initial value at $25^{\circ} \mathrm{C}$. The effect of temperature was widely describes in a previous paper [7]. The temperature provokes a double effect: an activation of the viscoelastic properties and of the stiffering phenomenon and a decrease in rigidity and endurance, attributed to thermal degradation of the cellular walls, at temperatures between $150^{\circ} \mathrm{C}$ and $180^{\circ} \mathrm{C}$ (Fig. 2). 


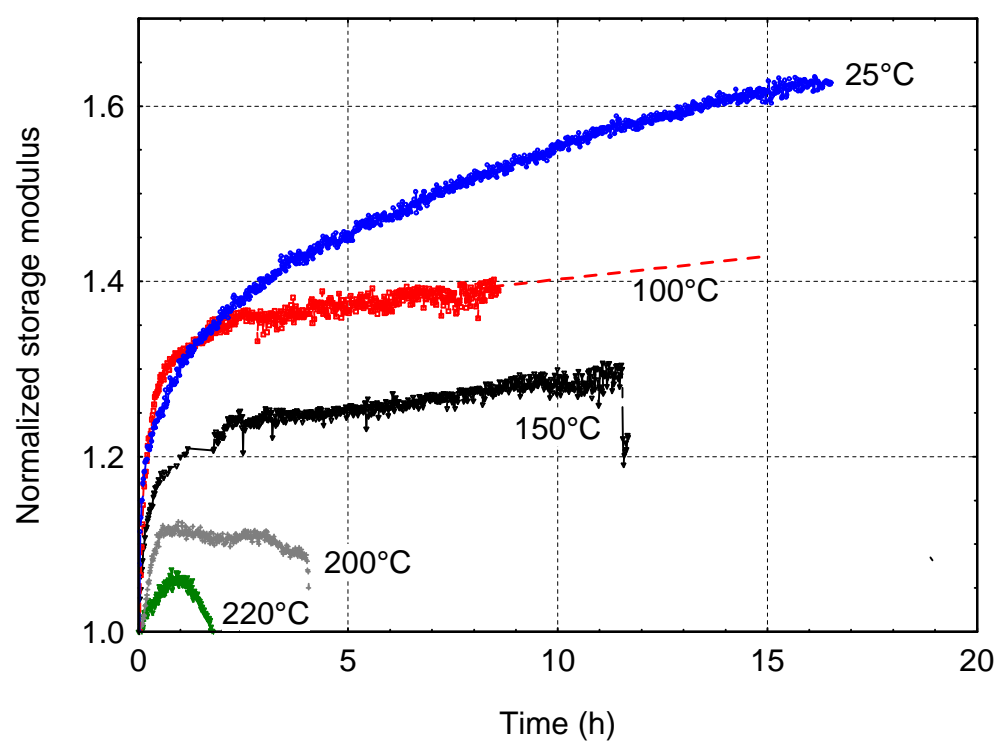

Fig. 2. Normalized storage modulus versus time at different plateau temperature, loading frequency: $1 \mathrm{~Hz}$ (bundle of hemp fibres) - Ambient humidity

The loading rate directly affects the stiffering phenomenon (Fig. 3). So, it is possible to affirm that this surprising phenomenon is time-dependant. Effectively, in the considered frequency range, the higher the frequency is, the higher the reinforcement of the rigidity after a same number of cycles is. For example, after 1000 cycles, the storage modulus is about1.3 times higher than the initial value for a solicitation frequency of $1 \mathrm{~Hz}$ while for $0.1 \mathrm{~Hz}$ the storage modulus is only 1.1 times higher. After a definite time of cycling, the fibre was unloaded during $15 \mathrm{~min}$. It clearly comes out that the stiffness increase due to cycling is a partially reversible phenomenon. The effect of the loading rate confirms the involvement of viscoelastic mechanisms in the stiffering phenomenon. This behaviour could be compared in a way of the thixotropic behaviour of some fluid, which show a timedependant change in viscosity, having a reduced viscosity when stress is applied. It is the same for the natural fibres, their viscosity decreases when periodic stress is applied (Fig. 3b.).

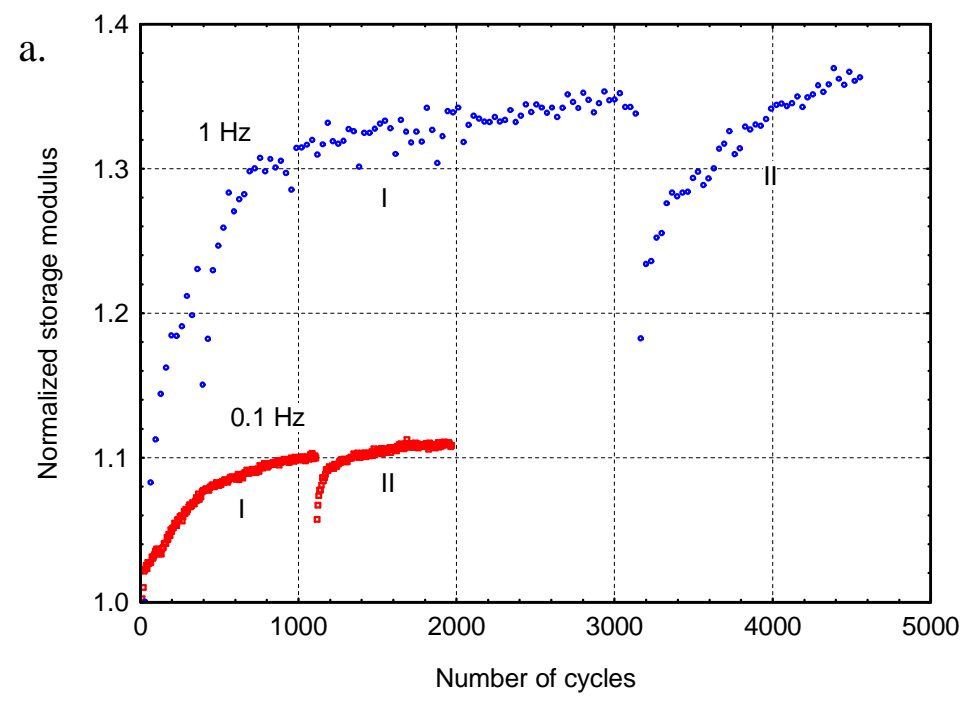




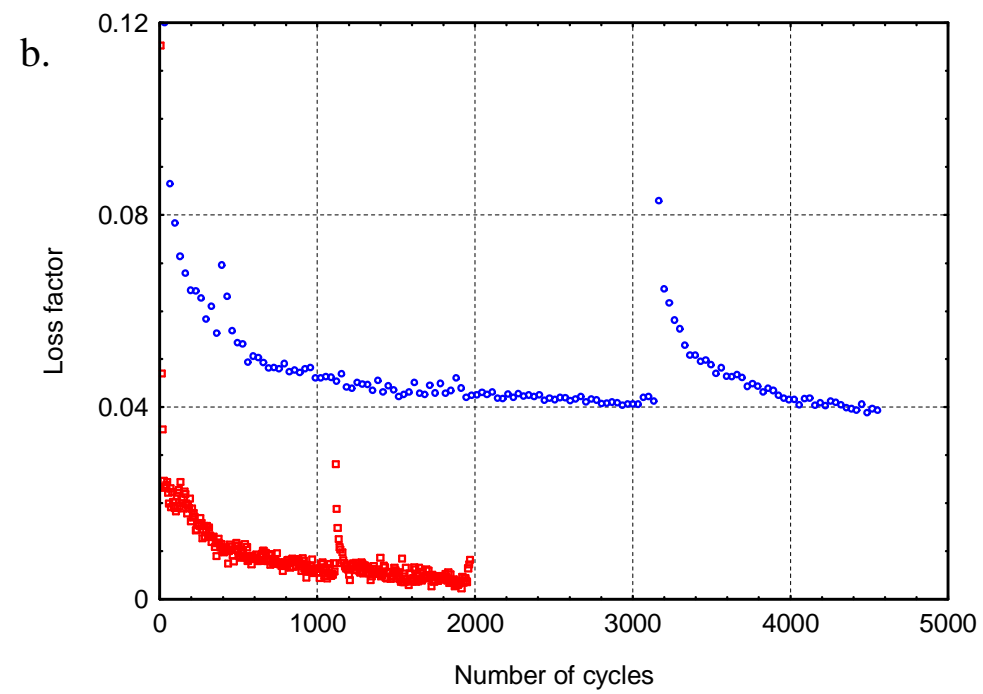

Fig. 3. Normalized storage modulus (a.) and loss factor (b.) versus number of cycles at 2 frequencies at room temperature. (I and II: 2 stages of loading separated by 15 min of unloading)

It is well known that the relative humidity directly affects the tensile properties of natural fibres [3]. According to Baley [4], the strength usually increases with moisture content, whereas the Young's modulus decreases as water is absorbed. In this work, we propose to investigate the effect of moisture content on the stiffering effect under cyclic loading. The control of humidity in a chamber of DMA is not straightforward and requires a high performance relative humidity generator.

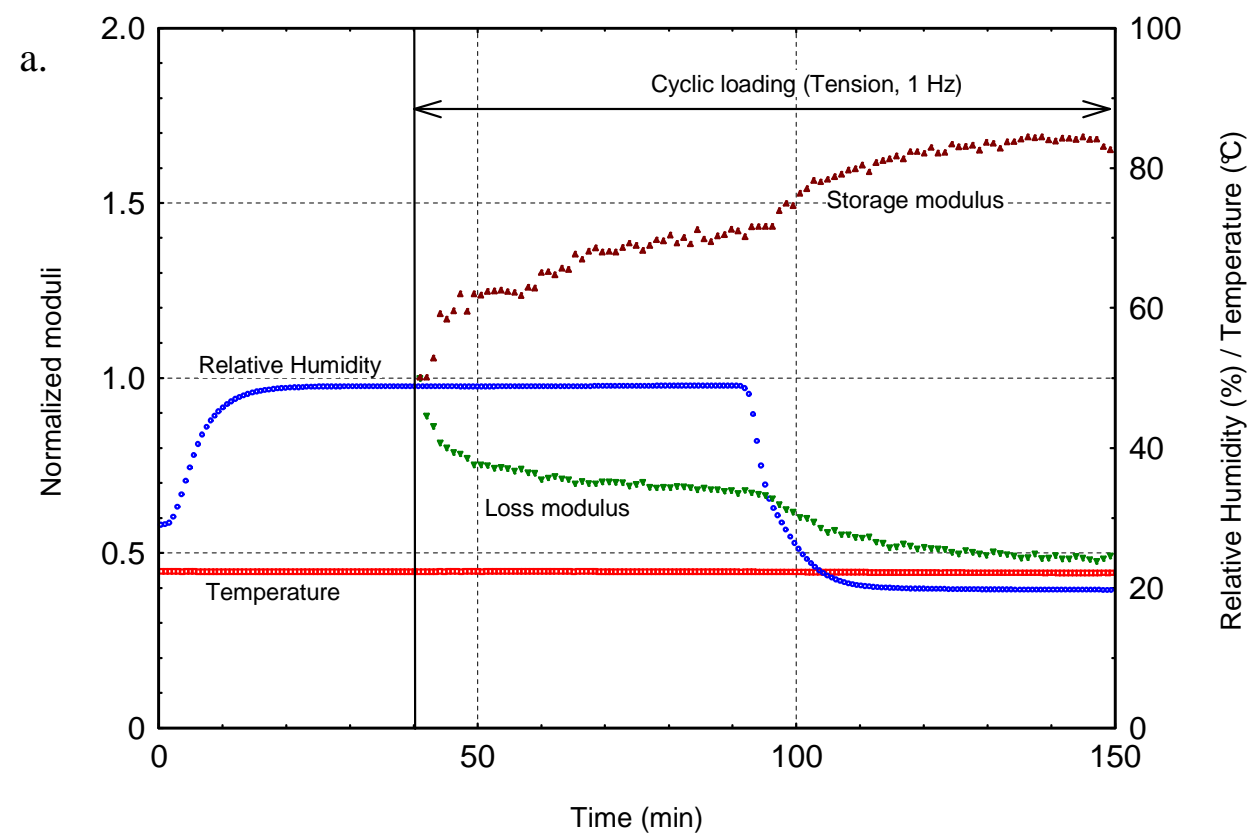




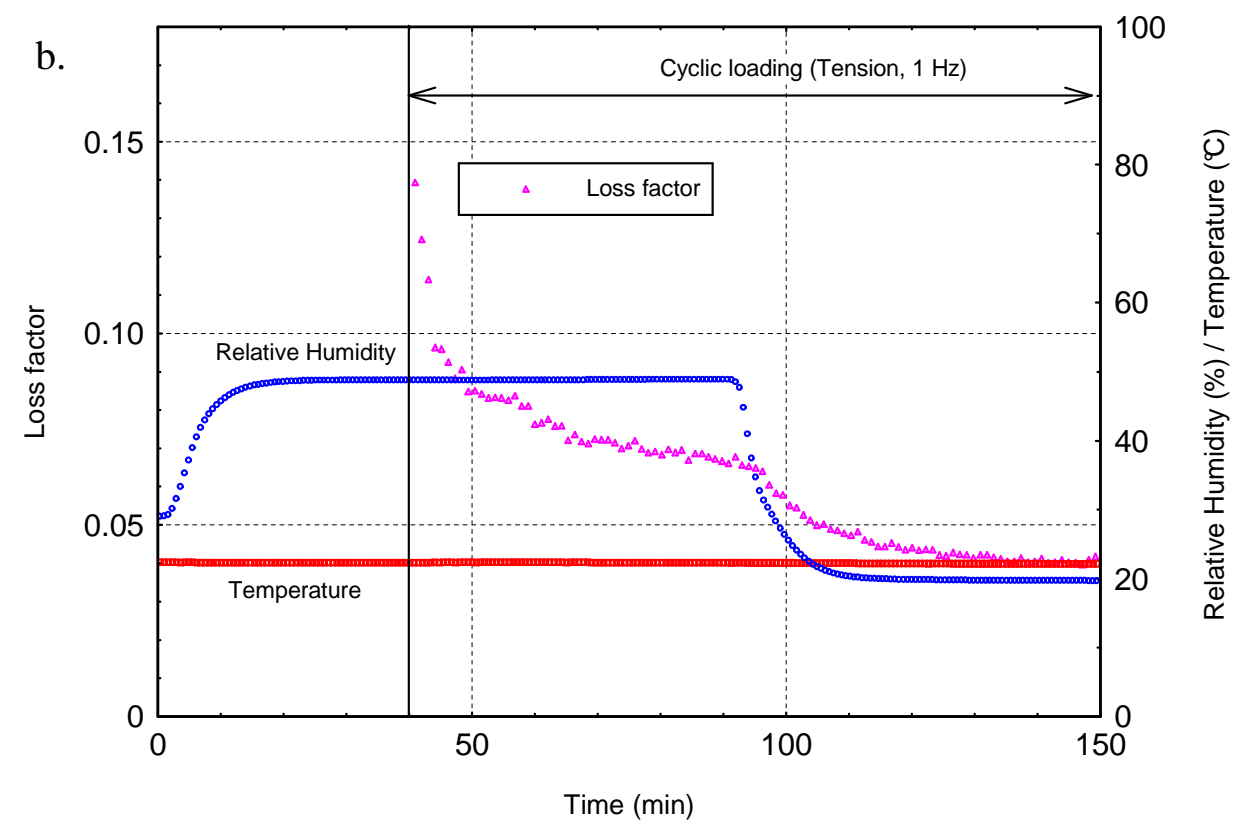

Fig. 4. Normalized storage modulus (a.) and loss factor (b.) versus time. Effect on relative humidity variation. Loading frequency: $1 \mathrm{~Hz}$, Temperatre: $22^{\circ} \mathrm{C}$.

The measurement of the viscoelastic properties under cyclic loading and during isothermal drying allows important variation in rigidity and viscosity to be underlined. The decrease in relative humidity from $50 \%$ to $10 \%$ involves a rising of the storage modulus and a decrease of the loss modulus and loss factor. This result is in accordance with the data collected on wood and wood components by numerous authors from long time [9, 11-14] and confirms the plasticizing effect of water on natural fibres.

\section{Conclusion and outlooks}

The application of cyclic loading on hemp fibres involves an increase in stiffness and a decrease in viscosity of natural fibre until a constant value is reached after a certain number of cycles (accommodation). This particular phenomenon is highly affected by the loading rate, the temperature and the moisture content. The stiffering of natural fibre under cycling loading:

- $\quad$ is altered by temperature higher than $150^{\circ} \mathrm{C}$,

- increases with loading rate,

- increases with drying.

It suggests the contribution of viscoelastic and mechano-sorptive phenomena. Recently, X-ray diffraction wide angle (WAXS) technique was used to determine the crystalline structure of cellulose and try to evaluate the microfibril angle (MFA). The first results are encouraging and seem to confirm the hypothesis of microfibrils rearrangements in the cell wall under cyclic loading The impact of this original behaviour of natural fibre on the global behaviour of organic matrix composite reinforced with natural fibres should also be queried.

\section{References}

1. Y. Xue, Y. Du, S. Elder, K. Wang, J. Zhang, Compos: Part B 40(3), 189-196 (2008)

2. J. Gassan, A.K. Bledzki, J Appl Polym Sci 82(6), 1417-1422 (2001) 
3. G.C. Davies, D.M. Bruce, Text Res J. 68(9), 623-629 (1998)

4. C. Baley, Compos: Part A 33, 939-948 (2002)

5. F.A. Silva, N. Chawla, R.D. Toledo Filho, Comp Sci Tech 68, 3438-2443 (2008)

6. F.A. Silva, N. Chawla, R.D. Toledo Filho, Mat Sci Eng A 516, 90-95 (2009)

7. V. Placet, Compos: Part A 40, 1111-1118 (2009)

8. A. Nechwatal, KP Mieck, T Reussmann, Comp Sci Tech 63, 1273-1279 (2003)

9. V. Placet, J. Passard, P.Perré, J Mater Sci 43, 3210-3217 (2008)

10. C. Assor, V. Placet, B. Chabbert, A. Habrant, C. Lapierre, B. Pollet, P. Perré. J Agr Food Chem 57(15), 6830-6837 (2209)

11. L. Salmén. J Mater Sci 19, 3090-3096 (1984)

12. GM. Irvine. Tappi J 67(5), 118-121 (1984)

13. SS. Kelley, TG. Rials, WG. Glasser. J Mater Sci 22, 617-624 (1987)

14. CA. Lenth, FA Kamke. Wood Fiber Sci 33(3), $492-507$ (2001) 